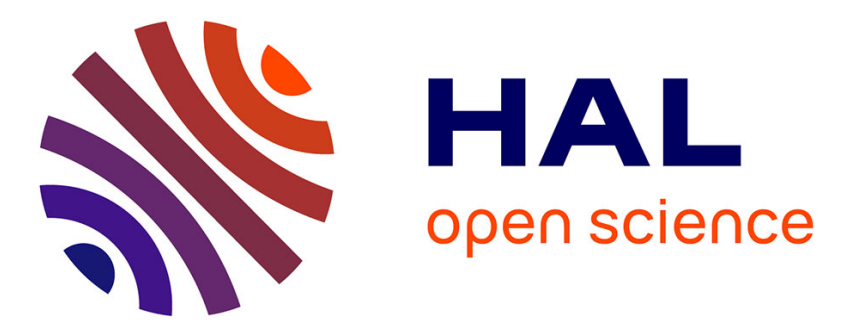

\title{
Corticospinal control of the thumb-index grip depends on precision of force control: a transcranial magnetic stimulation and functional magnetic resonance imagery study in humans \\ M Bonnard, C Galléa, J B de Graaf, J Pailhous
}

\section{To cite this version:}

M Bonnard, C Galléa, J B de Graaf, J Pailhous. Corticospinal control of the thumb-index grip depends on precision of force control: a transcranial magnetic stimulation and functional magnetic resonance imagery study in humans. European Journal of Neuroscience, 2007, 25, pp.872 - 880. 10.1111/j.14609568.2007.05320.x . hal-01453871

\author{
HAL Id: hal-01453871 \\ https://hal.science/hal-01453871
}

Submitted on 22 Nov 2017

HAL is a multi-disciplinary open access archive for the deposit and dissemination of scientific research documents, whether they are published or not. The documents may come from teaching and research institutions in France or abroad, or from public or private research centers.
L'archive ouverte pluridisciplinaire HAL, est destinée au dépôt et à la diffusion de documents scientifiques de niveau recherche, publiés ou non, émanant des établissements d'enseignement et de recherche français ou étrangers, des laboratoires publics ou privés. 


\title{
Corticospinal control of the thumb-index grip depends on precision of force control: a transcranial magnetic stimulation and functional magnetic resonance imagery study in humans
}

\author{
M. Bonnard, C. Galléa, J. B. De Graaf and J. Pailhous \\ Mediterranean Institute of Cognitive Neuroscience, UMR 6193, CNRS-GLM, Université d'Aix-Marseille, 31 chemin Joseph Aiguier, \\ 13402 Marseille, Cedex 09, France
}

Keywords: functional magnetic resonance imagery, precision grip, transcranial magnetic stimulation

\begin{abstract}
The corticospinal system (CS) is well known to be of major importance for controlling the thumb-index grip, in particular for force grading. However, for a given force level, the way in which the involvement of this system could vary with increasing demands on precise force control is not well-known. Using transcranial magnetic stimulation and functional magnetic resonance imagery, the present experiments investigated whether increasing the precision demands while keeping the averaged force level similar during an isometric dynamic low-force control task, involving the thumb-index grip, does affect the corticospinal excitability to the thumb-index muscles and the activation of the motor cortices, primary and non-primary (supplementary motor area, dorsal and ventral premotor and in the contralateral area), at the origin of the CS. With transcranial magnetic stimulation, we showed that, when precision demands increased, the CS excitability increased to either the first dorsal interosseus or the opponens pollicis, and never to both, for similar ongoing electromyographic activation patterns of these muscles. With functional magnetic resonance imagery, we demonstrated that, for the same averaged force level, the amplitude of blood oxygen level-dependent signal increased in relation to the precision demands in the hand area of the contralateral primary motor cortex in the contralateral supplementary motor area, ventral and dorsal premotor area. Together these results show that, during the course of force generation, the CS integrates online top-down information to precisely fit the motor output to the task's constraints and that its multiple cortical origins are involved in this process, with the ventral premotor area appearing to have a special role.
\end{abstract}

\section{Introduction}

The corticospinal system (CS) is known to be of major importance for organizing dexterous hand movements (Porter \& Lemon, 1993), in particular the precision grip, an ability that has developed in primates ensuring the manipulation of small objects with the tip of the thumb and fingers (Muir \& Lemon, 1983; Maier et al., 1993; Armand et al., 1996; Nakajima et al., 2000). In humans, a high level of dexterity can be obtained in actions such as writing, painting and surgical gestures. These activities continuously require a precise control of small dynamic forces exerted over the objects. It is unknown whether, for a given force, these high-precision (HP) demands do affect the involvement of the CS in thumb-index grip control (indeed, the precision of force control has rarely been isolated from other influencing factors, such as force magnitude and type of task). This is the core of this study. Such results would demonstrate the involvement of the CS in the precision of motor control during the executive phase.

In human beings, the CS control of precision grip has been investigated with transcranial magnetic stimulation (TMS). Several authors reported that, for similar electromyographic (EMG) back-

Correspondence: Dr Mireille Bonnard, as above.

E-mail: bonnard@incm.cnrs-mrs.fr

Received 14 March 2006, revised 14 November 2006, accepted 27 November 2006 grounds, the amplitude of motor evoked potentials (MEPs) in the first dorsal interosseus (FDI) is greater during a precision grip than in power grip or simple manual tasks (Schieppati et al., 1996; Hasegawa et al., 2001). They proposed therefore that the CS excitability increases with the precision level required by the task (Flament et al., 1993; Lemon et al., 1995; Schieppati et al., 1996). However, these studies compared different tasks, involving a different number of muscles or different muscle synergies, factors known to influence CS excitability (Aimonetti \& Nielsen, 2002; Devanne et al., 2002). Therefore, it is quite impossible to conclude that the required precision level is responsible for the observed modulations of MEPs in precision grip.

Concerning brain activation, differences were reported in the activation of the primary and non-primary motor cortices during fine control of precision grip. Using functional magnetic resonance imagery (fMRI), it was shown that the contralateral primary sensory motor cortex (SM1), dorsal premotor area (PMd), ventral premotor area (PMv) and bilateral supplementary motor area (SMA) were more activated during a gentle (with lower force magnitude) than a normal precision grip (Kuhtz-Buschbeck et al., 2001). In this study, the different motor control strategies (automatically vs. voluntarily controlled grip force) might explain the observed differences. Recently, in order to identify whether demands for precise control of the force affect brain activation, we introduced a task design in 
which the task was the same (voluntary visually-guided forceproduction task) with only the precision demands changing (Galléa et al., 2005). When contrasting HP and low-precision (LP) demands, we observed an increased blood oxygen level-dependent (BOLD) activation in the bilateral primary motor cortex (M1), PMv, PMd and SMA (Galléa et al., 2005); however, the analysis allowed neither the isolation of the hand area in M1 (M1-Ha) nor the quantification of BOLD signal changes.

In this experiment, we used TMS and fMRI to investigate the global CS excitability and BOLD signal changes in the area at the origin of the CS tract (M1-Ha, SMA, PMv and PMd) as the precision of grip control increased.

\section{Materials and methods}

\section{Transcranial magnetic stimulation experiment}

\section{Subjects}

Eight normal right-handed volunteers (three females and five males aged from 22 to 32 years) participated in this study. They were screened for magnetic field compatibility during a medical visit. All subjects gave written informed consent and were paid for their participation. The experiment was approved by the ethics committee (CCPPRB Marseille 1, DGS 990460) and was in accordance with the declaration of Helsinki. Prior to the experiment, all subjects were familiarized with the TMS and with the experimental task to ensure stable performance.

\section{Protocol and experimental design}

Subjects were seated in a chair, their right forearm immobilized horizontally in a semiprone position with their wrist in a comfortable cast. Throughout the experiment the wrist/hand configuration was secured by a polystyrene device (see Fig. 1). Subjects held a force transducer (Entran model ELPM-T1), whose thickness was $2.5 \mathrm{~cm}$, between the thumb and index finger of the right hand. The transducer and the two fingers rested on a support so that they were maintained in an approximate horizontal plane. The other fingers were relaxed over the polystyrene device and did not take part in the task. This device allowed us to secure the position of the hand and digits, known to greatly influence the EMG patterns.

The experiment was controlled with the help of a LABVIEW program. The subjects had to modulate their precision grip forces to track a force curve passing on a computer screen with a cursor whose vertical position corresponded to their instantaneous fingertip force (see Fig. 1). The cursor, permanently placed at the centre of the screen in the horizontal dimension, moved up and down with the subject increasing or decreasing the squeezing force. At any time, $3.5 \mathrm{~s}$ of the force curve was visible on both sides of the cursor on the screen, meaning that the subject could anticipate the force control. The subjects were told that, sometimes, TMS would be applied that could eventually induce small perturbations in the force output and move the cursor outside the instructed force curve. If so, they were asked to return comfortably to their curve-tracking task each time the TMS pulse moved the cursor away from the required force curve and not to voluntarily intervene to anticipate the compensation of these potential TMS-induced force perturbations, as we know they can. Indeed, previous experiments showed that, when instructed to do so, subjects can cognitively prepare themselves to resist a TMS-induced movement perturbation by selective modulations of CS excitability (Bonnard et al., 2003; see Bonnard et al., 2004 for a review).

The unpredictable force curve (see Fig. 1) to track lasted $120 \mathrm{~s}$ and was generated prior to the experiment as follows. A set of points was randomly generated between 3 and $7 \mathrm{~N}$ with a mean of $5 \mathrm{~N}$. This pattern of points was filtered at $4 \mathrm{~Hz}$ (second order low-pass Butterworth filter) resulting in a smooth curve of $5 \pm 1.55 \mathrm{~N}$. We then introduced (18 times) a short portion of the increasing part of a sinusoid into the smooth curve. This portion allowed us to apply TMS in comparable situations (i.e. at the same force level with a similar rate of force increase). The length of this sinusoidal portion varied and was
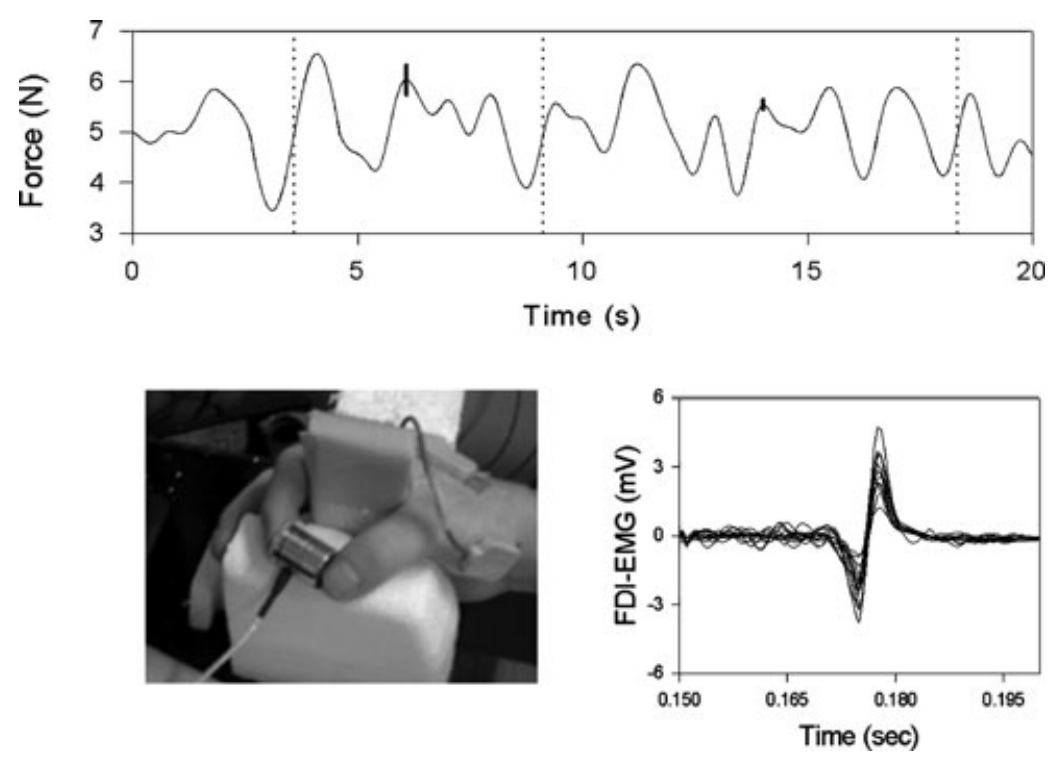

FIG. 1. (Top) $20 \mathrm{~s}$ of the instructed force curve-to-track with the small (high-precision condition) or large (low-precision condition) cursor. The cursor appeared at the centre of the screen during the experiment but in the figure the large and small cursors are represented by bold vertical lines in the left and right part of the figure, respectively. The dotted vertical lines indicate the times when transcranial magnetic stimulation (TMS) was applied. (Bottom left) Photograph of the force transducer and experimental set-up used in the TMS experiment. (Right) Recordings of the raw electromyographic (EMG) activity (mV) of the first dorsal interosseus (FDI) following the TMS pulse corresponding to the short-latency component of the motor evoked potentials (MEPs). Several MEPs have been superimposed to show their stability and reproducibility. 
adapted manually to the smoothed curve giving the impression of an unpredictable continuous curve.

We varied the precision level by using two cursor sizes corresponding to 0.2 and $0.6 \mathrm{~N}$. As determined by behavioural pretests, $0.2 \mathrm{~N}$ represented a HP demand and $0.6 \mathrm{~N}$ represented a LP demand. Prior to the experiment, the subjects were trained during two to four sessions on the tracking task so that they obtained an adequate level of performance (with a cursor lying on the force curve at least $60 \%$ of time).

The experiment consisted of two sessions and each session comprised two trials (each lasting $120 \mathrm{~s}$ ), one for each cursor size. The presentation order of the cursor sizes in each session was counterbalanced between the subjects. Throughout the experiment, the experimenter evaluated the performance and encouraged the subjects. Moreover, the experimenter monitored the EMG patterns. In the case of any visible abnormal change in the coactivation level, the subject was asked to come back to the original level. The subjects rested for 2 min between trials and between sessions.

\section{Magnetic stimulation and recording techniques}

Transcranial magnetic stimuli were applied over the scalp to the left motor cortex with a Magstim-200 stimulator (maximum magnetic field strength of $1.7 \mathrm{~T}$, Magstim, Dyfed, UK) using a focal doublecone coil (outside diameter $110 \mathrm{~mm}$, with an induced current flowing in the posterior-anterior direction). The Magstim-200 stimulator produced a damped, monophasic electric field of about $150 \mu$ s in duration. The subjects wore a latex swimming cap and surface markings were then drawn onto the cap to serve as a reference grid against which the coil was positioned. The coil was positioned over the left hemisphere, such that the central point between the loops was about $2 \mathrm{~cm}$ anterior and $5 \mathrm{~cm}$ lateral to the vertex $(\mathrm{Cz})$ that represents the most probable location of the cortical neurones projecting on the contralateral intrinsic hand muscles in M1. For each subject, fine adjustments of the coil position were made at the beginning of the experiments to identify the optimal locations for evoking MEPs in both muscles under investigation [FDI and opponens pollicis (OP)]. Stable fixation of the coil on the subject's head was then achieved by means of straps surrounding the head-coil system. The weight of the coil and its cable was relieved by attaching the whole to the ceiling with an elastic cord. Throughout the experiment, one experimenter further secured the fixation of the coil over the subject's head by apposing his hands at the border of the coil touching the head of the subject. This procedure ensured stable responses (see Fig. 1).

Bipolar EMG recordings from the OP and FDI were obtained from DE-2.3 signal conditioning electrodes (Delsys) using a parallel-bar contact placed over the belly of the respective muscles. The length of each electrode was $2 \mathrm{~cm}$ and the distance between the two parallel-bar contacts was $1 \mathrm{~cm}$. The signal from the electrodes was filtered $(20-450 \mathrm{~Hz})$ and preamplified with an isolated preamplifier (gain 1000) mounted into the electrode. The ground reference electrode was placed on the styloid process of the ulna. The EMG signals were then amplified with appropriate gains. The EMG signals, force transducer signal, instructed force pattern and stimulation trigger were recorded simultaneously with a sampling frequency of $2 \mathrm{kHz}$ using a multitask, real-time ADWinPro system (Keithley).

We determined the threshold to observe an MEP in the FDI when the subject was relaxed with the hand in a rest position (intensity of stimulation for which a potential of $50 \mu \mathrm{V}$ was evoked in four out of eight trials). Throughout the experiment, the intensity of the stimulation was kept constant, with a value of $0.85 \times$ this rest motor threshold (around $25 \%$ of the maximal intensity of the stimulator capacity). This intensity was chosen because it was found to evoke clear motor potentials in the muscles under study without generating too much muscle force, which could have perturbed the force tracking task. TMS triggering was controlled with the multitask, real-time ADWinPro system (Keithley). Based on the force transducer signal, the software controlled the stimulation occurrence when the subjects increased their force (positive slope, see Fig. 1) and passed the values of $5 \mathrm{~N}$ in the periods of time when we had inserted the sinusoidal portion (as previously described). The interstimulus interval was varied from $4 \mathrm{~s}$ (minimal value) to $9 \mathrm{~s}$ (maximal value) in order to be unpredictable for the subjects. In each trial of $120 \mathrm{~s}$, there was an average of 18 stimulations.

\section{Data reduction and statistical analysis}

Isometric force output. The data were processed off-line. The force signal was analysed as follows. Firstly, in order to verify the stability of the force production, we averaged the force signal for each experimental condition (LP and HP) over the whole duration of the trial. Secondly, to verify whether the participants modified the precision of their force control as a function of their cursor size, we calculated the mean distance between the midpoint of the cursor and the force imposed by the curve-to-track in each condition. The adaptation to the HP demand corresponds to less distance between the midpoint of the cursor and the force curve-to-track. Finally, we calculated the percentage of time spent by the cursor on the curve. For all of these measurements, we excluded each post-TMS time period for $500 \mathrm{~ms}$ for caution. These variables (averaged force, mean distance between the cursor midpoint and force-to-track, and percentage of time spent by the cursor on the curve) were subjected to repeated measures ANOVAs with two precision conditions (LP and $\mathrm{HP}$ ) as repeated measures factor. The level of significance was set at 0.05 .

Motor evoked potentials in first dorsal interosseus and opponens pollicis. For each time period during which TMS was applied, the following parameters were then calculated: the background EMG (based on the mean rectified EMG signal over $100 \mathrm{~ms}$ preceding the stimulation artefact) and the peak-to-peak MEP for the recorded muscles (based on the difference between the lowest and highest values of the raw EMG signals within a time window from 20 to $35 \mathrm{~ms}$ after the stimulation artefact) for each of these muscles. For each muscle, MEPs were compared according to the precision demands. However, as MEPs change as a function of the background EMG in each subject, the mean size of the MEPs was compared only for trials explicitly selected so that the mean background EMG was not statistically different in the two tasks. The criterion for MEP selection for the statistical analyses was that, for each subject, independently for each muscle, the background EMG fell within the common range for the two precision levels (Schieppati et al., 1996). Given the different number of selected trials $(15<N>36)$ in each condition for each subject, statistical analyses were conducted separately in each subject over the whole data set selected for each dependent variable, with the factor precision (LP and HP) using a Student's unpaired $t$-test (to take into account the maximum number of observations that were different in each condition due to the selection process). In each subject, this allowed the isolation of one particular muscle to which the CS excitability increased with precision, i.e. a 'controlling muscle'. To analyse the whole group, a non-parametric Wilcoxon test for paired data was then conducted over the MEPs of the 'controlling' muscle of all subjects. The significance threshold was kept constant throughout the statistical analyses $(P=0.05)$. 


\section{Functional magnetic resonance imagery experiment}

Subjects

Eleven normal right-handed volunteers (three females and eight males aged from 22 to 32 years) participated in the fMRI study. Seven of them had participated in the TMS study. They were screened for fMRI compatibility during a medical visit. All subjects gave written informed consent and were paid for their participation. The experiment was approved by the public assistance of Paris Hospitals and the local ethics committee (CCPPRB RBM 01-04) and was in accordance with the declaration of Helsinki.

\section{Protocol and experimental design}

Blood oxygen level-dependent activity was measured during execution of the same isometric dynamic task as in the TMS experiment. This fMRI analysis is part of a larger study on the influence of precision of force control on BOLD activation, in which we varied the precision level by using five cursor sizes. We refer to Galléa et al. (2005) for more details. Here, we only report BOLD activation in the M1-Ha, SMA, PMv and PMd (corresponding to the multiple origins of the CS) for the two cursor sizes corresponding to those used in the TMS experiment (equal to the smallest and largest cursors in our previous fMRI experiment, Galléa et al., 2005). The design was adapted to the constraints of fMRI. Subjects had a circular force transducer (metallized polycarbonate, compatible with the magnetic environment) clutched between two pieces of rigid elastomer between the thumb and index finger of the right hand. Other fingers were bent over the palm of the hand and did not participate in the task. The grip object was maintained only by the two fingers in an isometric condition. Laid on the fMRI bed, the subject's right wrist and forearm were fixed on the body with the hand near their groin to avoid perturbations caused by breathing movements.

The curve that the subjects had to follow was similar to that used in the TMS experiment. We did not, however, introduce the portion of sinusoidal waveform during which TMS was applied into the smooth curve. We varied the precision level by using five cursor sizes. Each cursor size was presented for $25 \mathrm{~s}$. Each time that a transition between two cursors of different sizes occurred, the force curve was frozen for $1.3 \mathrm{~s}$ on the screen. Each cursor size appeared twice in a session. The total experiment consisted of five sessions (or runs), each lasting $5 \mathrm{~min}$. Therefore, a total of 10 trials per cursor were performed. The subjects rested for 2 min between every two sessions. The order of the conditions was counterbalanced between the sessions and subjects. The same cursor was never presented twice in a row.

The subjects were trained in the force tracking task before the fMRI acquisition in circumstances imitating as much as possible those of the fMRI acquisition (i.e. horizontal position, tunnel simulation and dark environment). This training ensured stable performance and allowed the subjects to become familiar with the experimental setting. The whole experiment was practiced 1 week before fMRI acquisition and one session was again practiced in the fMRI machine just before the start of the first session with the specific noise of the fMRI acquisition. During scanning, the curve to be tracked and the cursor were presented on a computer screen reflected in mirror glasses fixed on the subject's head.

\section{Data acquisition}

Isometric force output. The grip forces exerted over the force transducer were sampled at a frequency of $100 \mathrm{~Hz}$ by a Labview program and saved on a hard disk for off-line analysis.

Functional magnetic resonance imagery data. Imaging was carried out with a 3-Tesla whole-body Brucker Imager (MEDSPEC 30/80
AVANCE, centre IRMf, Marseille, France). For all subjects, the experience began with the acquisition of high-resolution structural T1weighted images for anatomical coregistration (15 min, voxel size $1 \times 0.75 \times 1.22 \mathrm{~mm}$ ). Functional imaging was then performed in five sessions. The functional images were acquired using a $\mathrm{T} 2 *$-weighted echo-planar sequence at 20 axial slices (repetition time $1.66 \mathrm{~s}$, interleaved acquisition, slice thickness $3 \mathrm{~mm}$, interslice gap $1 \mathrm{~mm}$, $64 \times 64$ matrix of $3 \times 3-\mathrm{mm}$ voxels). The slices covered the upper brain and contained the frontal, parietal and almost all of the occipital lobes. The slices were acquired parallel to the anterior commissureposterior commissure plane. A total of 790 volumes were acquired and submitted for analysis.

\section{Analysis}

Isometric output force. The force signals were analysed in the same way as in the TMS experiment.

Functional magnetic resonance imagery data. We defined several regions of interest (ROIs) on the basis of the individual anatomical data of each subject. Figure 2 shows the left M1-Ha, SMA, PMd and $\mathrm{PMv}$ for one subject.

Concerning M1, the superior margin of the left hemisphere was taken as its superior border, M1-Ha being restricted to the lateral extension on the tau or omega curvature of the central sulcus (Yousry et al., 1997). Inferiorly, we have been careful not to consider the whole depth of the central sulcus because of the uncertain delimitation between M1 (Brodmann area 4) and the primary sensory cortex (Brodmann area 3). Thus, we chose only the surface of the anterior bank of the central sulcus.

Brodmann area 6 is usually subdivided into a medial part (SMA) and a dorsolateral part (premotor area). For the medial part, SMA was delimited as follows: superiorly bounded by the upper border of the interhemispheric fissure and inferiorly bounded by the cingulated sulcus (Roland \& Zilles, 1996). Moreover, there is agreement that vertical planes intersecting the anterior commissure and posterior commissure constitute approximately the anterior and posterior borders of SMA (Geyer et al., 2000). In the dorsolateral part, the precentral gyrus, considered as the location of the premotor area, was divided in two parts relative to the perpendicular projection of the superior frontal sulcus (Rizzolatti et al., 1998). The PMd was defined superiorly by the border of SMA and inferiorly by the projection of the superior frontal sulcus. The PMv was defined in the superior part by the virtual prolongation of the superior frontal sulcus (perpendicular to the central sulcus) and in the inferior part by that of the inferior frontal sulcus.

We analysed the raw BOLD signal in each ROI in order to evaluate its variation with the precision demands. After a conventional preprocessing using SPM99 (http://www.fil.ion.ucl.ac.uk/spm/), the ROI analysis was performed with the freely available ROI toolbox MARSBAR (http://marsbar.sourceforge.net/). The mean signal (averaged over all voxels contained in the ROI) was extracted from each ROI volume for the whole duration of the experiment. Individual variations of the raw data were obtained for each condition for all subjects. For all of the individual data in each ROI, we corrected the linear drift occurring for each session with a detrend function of MATLAB. To cancel differences in BOLD signal between sessions and between subjects, the mean BOLD value over each session in each ROI was set to 180 (180 is a value often attributed to the grey matter signal considering that the whole brain mean signal is 100 , see http:// marsbar.sourceforge.net/). Considering the delay between the condition occurrence and the BOLD signal to be $6 \mathrm{~s}$, we shifted by this amount of time the time course of the BOLD signal with respect to the time course of the condition occurrence. The whole time series of the 


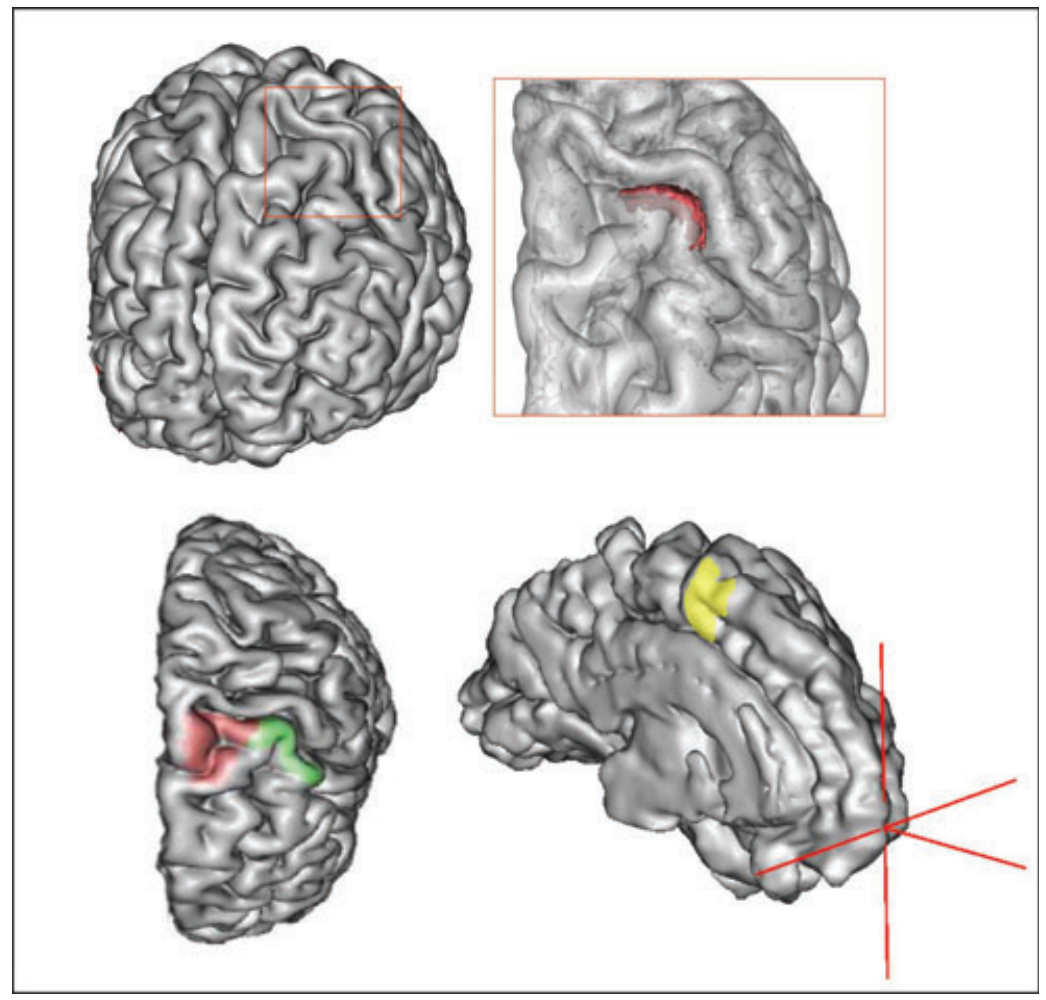

FIG. 2. (Top) Surface rendering of the brain of one subject with a magnification of the peri-rolandic region. The red area represents the region of interest that we identified as the hand area of the left primary motor cortex for this subject. (Bottom left) Top view of the left hemisphere showing the delimitation of the left ventral premotor area (green area) and left dorsal premotor area (red area). (Right) Medial view of the left hemisphere showing the supplementary motor area (yellow area). For anatomical delimitations, see the text.

BOLD signal was then cut into the successive conditions and the scan ending each condition was removed to exclude the task switch period. For the purpose of statistical analysis, for each subject, we averaged the time series over each trial for each condition (block of $25 \mathrm{~s}$ ). Here we only report the results for the two cursor size corresponding to those used in the TMS experiment. Results were expressed in terms of the amplitude of the BOLD signal coming from the raw data. For each ROI, the comparison between conditions was performed on this average with a repeated-measures ANOVA with two precision conditions, (LP, HP)*10 trials, as repeated-measures factors. In order to look for any pattern in brain activation, we then calculated the percent signal change in the HP compared with the LP condition in each ROI, and correlation analyses were conducted across these percent signal changes and the percent change in MEPs using the Kendall $\tau$ test. The level of significance was set at 0.05 for all of these analyses.

\section{Results}

\section{Transcranial magnetic stimulation experiment}

\section{Isometric force output}

The mean force produced during the task nicely fitted the required $5 \mathrm{~N}$ level in both LP and HP conditions and did not differ between conditions $\left(F_{1,7}=1.4, P>0.05\right)$. The mean distance between the midpoint of the cursor and the force imposed by the curve-to-track was found to decrease with higher precision demands [from $0.19 \pm 0.026 \mathrm{~N}$ in LP (mean $\pm \mathrm{SD}$ ) to $0.17 \pm 0.026 \mathrm{~N}$ in HP, $\left.F_{1,7}=43.5, P<0.05\right]$, which confirms the higher precision of the force control. The percentage of time spent by the cursor on the curve decreased with the increase of precision (from $98 \pm 1.4 \%$ in LP (mean $\pm \mathrm{SD}$ ) to $65 \pm 6.1 \%$ in HP), showing more errors with higher precision demands $\left(F_{1,7}=257.1, P<0.05\right)$.

\section{Prestimulus electromyographic background}

Figure 3 presents the mean initial EMG background (preceding the TMS pulse) observed, for each subject, in the FDI and OP for the selected trials for each experimental condition. None of the subjects showed any significant difference in initial EMG background with precision in either FDI or OP ( $P$ always $>0.05)$, which validates the selection of the analysed data.

\section{Motor evoked potentials in first dorsal interosseus and opponens pollicis}

Figure 3 shows, for each subject, the mean MEP size of FDI and OP observed for the selected trials for LP and HP demands. The two data sets were compared separately for each subject and this revealed the existence of two separate subgroups in the HP condition. The first included four subjects showing a selective increase in CS excitability to FDI with increasing precision demands $(P<0.01$ for each subject $)$ and the second included three other subjects who showed an increase in CS excitability to OP $(P<0.04$ for each subject). This selective increase in CS excitability to one muscle was consistent enough to result in a significant change in individual MEPs in one muscle and not in the other in seven of eight subjects.

The group analysis of the MEPs in the 'controlling' muscle (the particular muscle to which the CS excitability increased with precision for each subject) yielded a significant effect of precision over the MEPs of the controlled muscle $(P=0.012)$. 

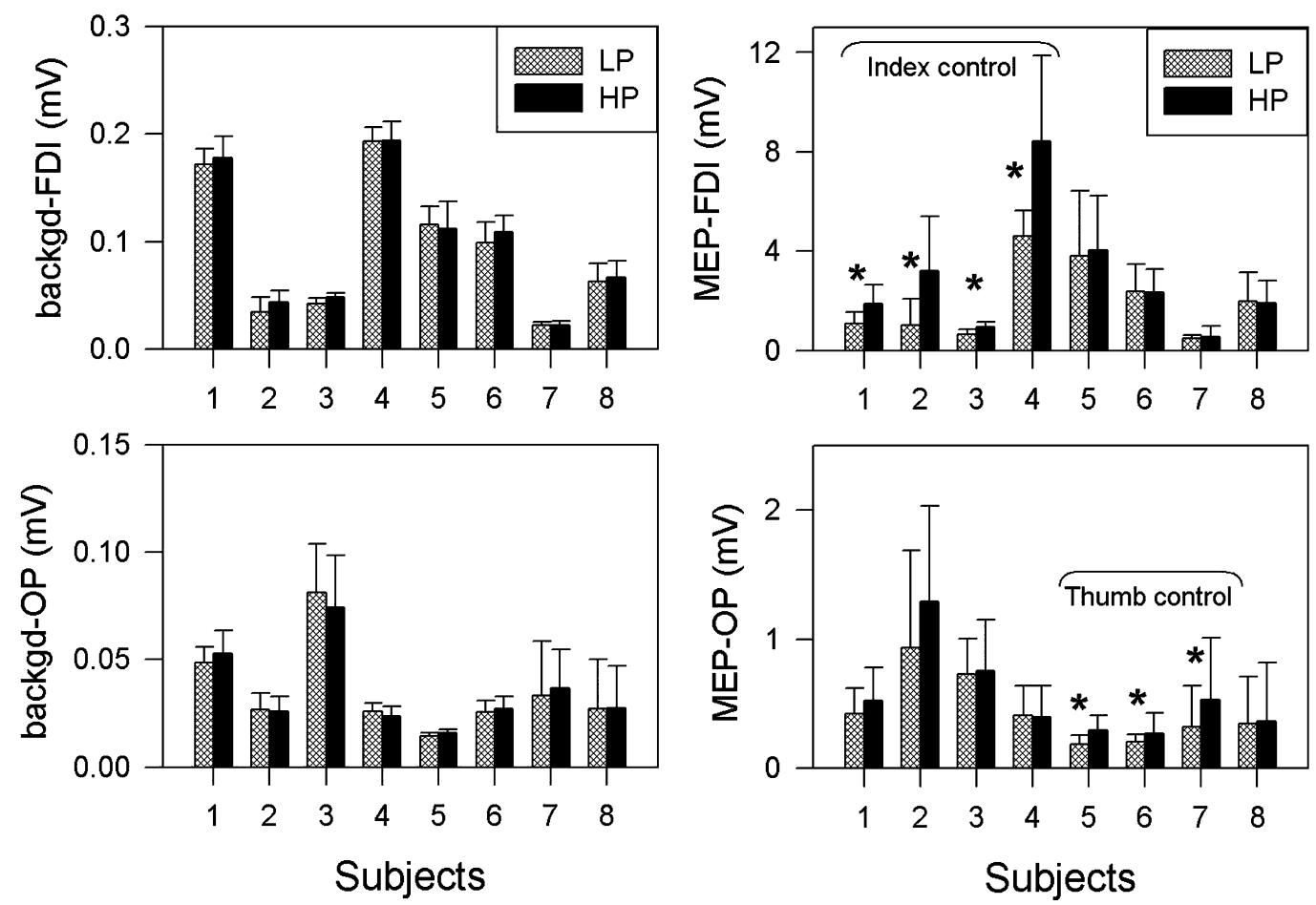

FIG. 3. Mean electromyographic backgrounds (left) and mean amplitude of motor evoked potentials (MEPs) (right) observed in the first dorsal interosseus (FDI) and opponens pollicis (OP) muscles for the selected trials of each subject in the low- (LP) and high-precision (HP) conditions. Each bar is the average of 15-36 trials and bars indicate SDs. *Significant difference between the two conditions for a given subject $(P<0.05)$.

Thus, the CS excitability was found to increase with precision demands and selectively in one muscle, representing one finger, but never in the two fingers at the same time. This argues in favour of one and the same strategy, i.e. engaging only one finger in precision control. It has to be noted that none of the subjects was aware of this particular strategy. To verify that these effects were not due to slight differences in EMG background, the same analyses were conducted on the MEP : background ratio and they confirmed all of the abovementioned results.

\section{Functional magnetic resonance imagery experiment}

\section{Isometric force output}

As in the TMS experiment, the mean force level performed by the subjects was $5 \mathrm{~N}$ for the two precision conditions, which corresponded to the mean force level of the curve-to-track. The average distance between the midpoint of the cursor and the force-to-track and the mean percentage of time spent on the curve were very similar to those observed in the TMS experiment.

Blood oxygen level-dependent activation in contralateral hand area in primary motor cortex, supplementary motor area and premotor area

Figure 4 presents the average BOLD signal in the left M1-Ha (LM1Ha) observed for the two precision levels in a typical subject plotted against time.

ANOVA performed on the BOLD signal of the different ROIs under study yielded a significant effect of precision level on BOLD activation of the LM1-Ha $\left(F_{1,10}=13.9, P<0.05\right)$, of the left SMA (LSMA) $\left(F_{1,10}=67.9, P<0.05\right)$ and of the left PMv (LPMv) $\left(F_{1,10}=28.9, \quad P<0.05\right)$ and left PMd (LPMd) $\left(F_{1,10}=32.9\right.$, $P<0.05)$. For the same averaged force produced, the activation of

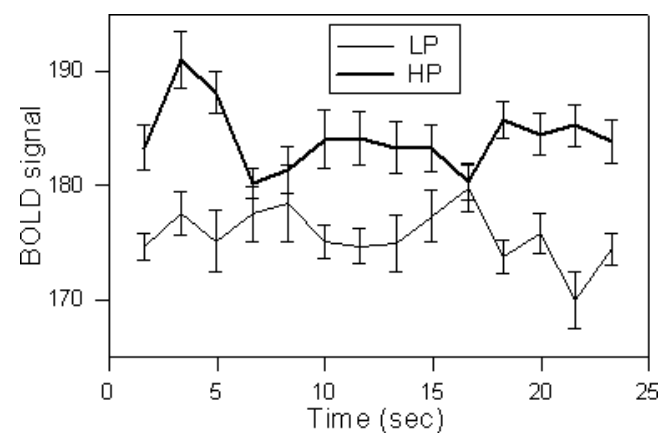

FIG. 4. Time course of the averaged blood oxygen level-dependent (BOLD) signal observed in the hand area of the primary motor cortex for a typical subject in the low- (LP) and high-precision (HP) conditions. Bars indicate SEs.

all of these areas was found to increase with the precision demand of the force control (from 3.8\% in LM1-Ha, $0.26 \%$ in LPMd, $0.25 \%$ in LPMv and $0.27 \%$ in LSMA), which corresponds to values reported in the literature for other motor tasks (Porro et al., 1996). Figure 5 presents the percent signal change observed between HP and LP conditions for each subject in LM1-Ha, LSMA, LPMv and LPMd.

Interestingly, correlation analyses yielded significant correlations between percent signal change in LPMv and LM1-Ha (Kendall $\tau=0.51, P<0.05)$, between LPMv and LSMA $(\tau=0.47, P<0.05)$, and between LPMv and LPMd $(\tau=0.69, P<0.05)$. Thus, the percent signal change in LPMv correlates with the signal change in all of the other ROIs, and no correlation was found between LM1-Ha, LSMA and LPMd. In order to find out whether any correlation could be observed between fMRI and TMS data, we also conducted correlation analyses across the percent BOLD signal changes 


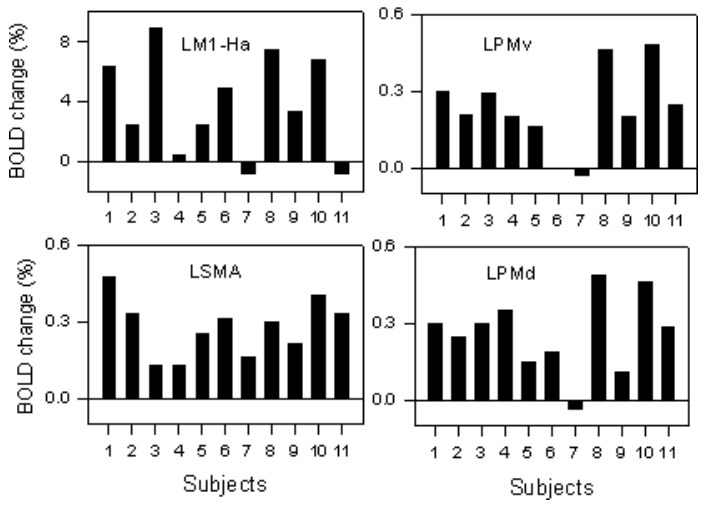

FIG. 5. Mean percent blood oxygen level-dependent (BOLD) signal change observed in the high-precision compared with the low-precision condition for each subject in the left hand area in primary motor cortex (LM1-Ha), left supplementary motor area (LSMA), left ventral premotor area (LPMv) and left dorsal premotor area (LPMd).

(observed in the HP compared with the LP condition) in the different ROIs under study and the percent change in CS excitability to the controlling muscle (the particular muscle to which the CS excitability increased with precision for each subject) for the seven subjects who took part in both the TMS and fMRI experiments. We did not find any significant correlation $(-0.3<\tau<0.06, P>0.05$ for all of the analyses).

\section{Discussion}

Two major results were obtained in these experiments by comparing the same isometric dynamic force tracking task performed with the thumb-index grip under different precision demands. The first is that, as shown by the TMS experiment, the MEPs in either one or the other (and never both) of the intrinsic hand muscles (OP or FDI, acting on the thumb or index finger, respectively) were significantly higher when the task's precision demands increased, although the EMG backgrounds of these muscles were similar for both conditions. The second is that, for the same averaged output force, BOLD activation increased with precision demands in the hand area of left contralateral M1, LSMA, LPMd and LPMv. Moreover, correlations were found between percent signal change in PMv and those in all of the other ROIs. However, no correlation was observed between fMRI and TMS data (i.e. between percent BOLD signal change and percent change in MEPs). We will now discuss these results in more detail.

\section{Effect of precision of force control on corticospinal system excitability}

As we used the same task in the present experiment, i.e. producing a similar dynamic force output with one and the same effector system (activated with similar EMG activation patterns), but under different precision demands, we were able to demonstrate the effect of the precision demands of force control on MEPs in seven of eight subjects. Several authors have already reported increased MEP amplitude during the precision grip in comparison with the power grip (Flament et al., 1993; Hasegawa et al., 2001; Tinazzi et al., 2003) or during different precision tasks compared with power tasks (Schieppati et al., 1996). However, as stated in the Introduction, in all of these studies the effect of precision could not be dissociated from the effect of the task itself because the task under comparison involved the production of different motor outputs. In this experiment, when precision of the thumb-index grip control increased, we showed an increase of MEP size in one or the other of the prime mover muscles (each moving a different finger), which strongly suggests that the corticospinal excitability to this muscle was increased.

It is interesting to note that, with increasing precision demands, the CS excitability never increased in the two fingers at the same time. Indeed, this suggests that, in order to fit the precision demands, different functions were attributed to the two fingers, one finger being especially engaged in the precise control, including precise force calibration and error corrections (thus its CS excitability was raised with increasing precision), whereas the other was devoted to a more postural or sustaining function (thus its CS excitability remained unaffected by increasing precision). In our experiment, as in the case of mental movement simulation (Rossi et al., 1998; Fadiga et al., 1999), important changes were observed not at the EMG level but in the MEPs. Indeed, although our EMG activation patterns were similar for the LP and HP demands, the MEPs allowed the identification of one finger as being selectively engaged in the precise force control and therefore revealed the control strategy of the subject, i.e. engaging only one finger in the precision control. The fact that the increase in CS excitability did not concern the same muscle in all of the subjects is probably due to individual strategies.

\section{Effect of precision of force control on the activation of motor cortices}

We used fMRI to investigate whether increasing precision demands on force control affects the global activation level of contralateral primary (M1-Ha) and non-primary (SMA, PMv and PMd) motor cortices. The results clearly showed an increase in regional cerebral blood flow in all of these ROIs when contrasting the production of the same isometric dynamic force under HP with respect to LP demands. Moreover, our TMS results strongly suggest that this increased regional cerebral blood flow as precision demands increased has a functional role, as we observed a selective increase in the CS excitability to one muscle.

Concerning the M1, several studies have already reported an increase in BOLD activation in SM1 during different precision grip tasks (Kuhtz-Buschbeck et al., 2001; Galléa et al., 2005) but this is the first time that observations have been made focusing on an individual anatomically defined ROI restricted to M1-Ha. This allows further interpretation of the increased activation in SM1 reported in the abovementioned studies. From their study, Kuhtz-Buschbeck et al. (2001) concluded that it was probably due to an increased attention to sensory (cutaneous and proprioceptive) information that could increase the activation of the primary sensory areas, which are in turn closely linked to motor areas. Without excluding this possibility of increased central processing of sensory information, our results clearly show that the BOLD activation in M1-Ha itself increased as the precision demands grew. This points out that dexterity requires not only a capability to process sensory information but also a capability to very precisely grade forces and rapidly correct the errors. The last point to be noted concerning this increased BOLD activation in M1-Ha with higher precision is that the fMRI data did not allow the identification of one finger as being selectively engaged in the precise force control, as did the TMS data. Indeed, we never observed that, in subjects with a thumb control strategy, the maximum difference (HP-LP) in signal was more lateral than in subjects with an index finger control strategy, which could have been predicted given the somatotopy of the sensorimotor homunculus. This could be explained by the wide overlap of somatotopic representations of the fingers (Schieber, 2001) and/or by the fact that, in our task, although one finger appeared to be the controlling one, both fingers were actively engaged. 
Concerning non-primary motor cortices (SMA, PMv and PMd), the increase in BOLD activation in SMA, PMv and PMd observed in the present study is similar to previous results obtained during different precision grip tasks (Kuhtz-Buschbeck et al., 2001; Galléa et al., 2005). However, the present study based on individual ROIs allows a complete and quantified description to be drawn of the observed change in these different cortical areas. Of particular interest was the fact that significant correlations were observed between the percent change in BOLD activation between ROIs that all involved LPMv, i.e. LPMv and LM1-Ha, LPMv and LPMd, and finally LPMv and LSMA. Thus, the percent signal change in LPMv is correlated with the percent signal change in all of the other primary and non-primary motor cortices. This strongly suggests that the contralateral PMv plays a particular role in the precise control of the exerted force. During movement preparation, the role of $\mathrm{PMv}$ in elaborating the motor commands for precise grasping has already been put forward by Davare et al. (2006). Indeed, using a transient virtual lesion of contralateral LPMv applied with TMS before movement onset, they observed disturbances of the sequential recruitment of intrinsic hand muscles. The present results strongly suggest that LPMv also plays a special role during movement execution, in the present case a precise control of the exerted force; whether or not this area has to be considered as a conductor of the orchestra remains to be established but it at least seems to be an important crossroads between cognitive and motor processes during movement execution. Interestingly from an anatomical point of view, Dum \& Strick (2005) found that PMv and PMd are the major sources of input (70\%) from the frontal lobe to the digit area in M1. Moreover, they observed that the projections of the digit area in SMA to PMv and PMd are stronger than the SMA projections to M1.

\section{General remarks on precise force control and corticospinal networks}

We were unsuccessful in observing any correlation between fMRI and TMS data. This means that the subjects who showed a strong increase of the MEPs in the HP condition (compared with the LP condition) did not exhibit a strong increase of the BOLD signal in the ROIs under study. It has to be noted that the lack of such a relationship between fMRI and TMS data has already been reported in a study of brain activity and TMS during simple and complex executed vs. imagined movements (Kuhtz-Buschbeck et al., 2003). Similarly, those authors did not find significant correlations between individual fMRI and TMS data, although increasing task complexity affected both the BOLD signal and corticospinal excitability. This is probably due to the different nature of these techniques revealing quite different processes (electrical vs. metabolic) that are obviously related but probably not that tightly.

The observed increased activation in primary and non-primary motor cortices and in CS excitability could be related to the motor attention required to send precisely calibrated motor commands to the effector system and to rapidly correct small errors. Indeed, in the present experiment, all of the tasks were voluntarily controlled (in contrast with the study of Kuhtz-Buschbeck et al., 2001) but more or less precisely. This attention hypothesis was brought to the fore by Johansen-Berg \& Matthews (2002) by removing attention to the motor task with a secondary task. In their study, they showed a decrease in BOLD activation of the right ipsilateral M1 (area 4p) and LSMA when a counting task took place in synchrony with a motor task. Therefore, they proposed that attention to movement modulates the activation of the brain network for motor control at multiple sites including M1. However, the study of Kristeva-Feige et al. (2002) showed that removing attention to movement by adding a secondary task does not involve the same cerebral mechanisms as adding attention towards the movement in order to fit precision demands. In our study, we reported an increased BOLD activation in the contralateral LM1-Ha, LPMv, LPMd and LSMA with increasing precision of the thumb-index grip control that could be interpreted as really being due to increased motor attention. Moreover, it has to be noted that this is not a non-specific attentional effect (global arousal) but rather a selective effect affecting one or other of the prime-mover muscles, moving either the thumb or index finger, as shown by our TMS results.

In conclusion, it was already known that the CS and its primary and non-primary cortical origins are places where cognitive processes related to movement are integrated during movement preparation, i.e off-line presetting (see, for reviews, Requin et al., 1991; Georgopoulos, 2000; Bonnard et al., 2004; Riehle, 2004). However, during movement execution, their role in on-line integration of cognitive and sensorimotor processes was far less clear. Indeed, most of the studies conducted during movement execution focused on relating their activation to the parameters of the motor output (direction, magnitude, duration and rate of change of torque or movement) rather than to motor control processes. Here, we showed that, for a similar mean force level, the precision demands on this force control affected both the corticospinal excitability and BOLD activation of the primary and non-primary motor cortices while the EMG activation patterns remained similar. Moreover, this integrative process was found to be specific to the finger engaged in the precise control. Together these results strongly suggest that, during the course of the movement, the CS allows integration of not only sensory and motor processes, as already well described (Evarts \& Fromm, 1978; Fromm, 1987), but also top-down information in order to precisely fit on-line the motor output to the task's demands. Moreover, we showed that the multiple origins of the CS tract (M1, SMA, PMd and PMv) are all involved in this process. How the interactions between these regions are organized remains to be established by future studies but PMv seems to play a special role in this concert.

\section{Acknowledgements}

This study was supported by the Centre National de la Recherche Scientifique and the French program 'Cognition and information processing'. The authors would like to thank Prof. A. Delarque who willingly assumed medical responsibility for the experiment.

\section{Abbreviations}

BOLD, blood oxygen level-dependent; CS, corticospinal system; EMG, electromyographic; FDI, first dorsal interosseus; fMRI, functional magnetic resonance imagery; HP, high-precision; LM1-Ha, hand area in left primary sensory motor cortex; LP, low-precision; LPMd, left dorsal premotor area; LPMv, left ventral premotor area; LSMA, left supplementary motor area; M1, primary motor cortex; M1-Ha, hand area in primary motor cortex; MEP, motor evoked potential; OP, opponens pollicis; PMd, dorsal premotor area; PMv, ventral premotor area; ROI, region of interest; SMA, supplementary motor area; SM1, primary sensory motor cortex; TMS, transcranial magnetic stimulation.

\section{References}

Aimonetti, J.M. \& Nielsen, J.B. (2002) Cortical excitability and motor task in man: an investigation of the wrist extensor motor area. Exp. Brain Res., 143, 431-439.

Armand, J., Olivier, E., Edgley, S.A. \& Lemon, R. (1996) The structure and function of the developing corticospinal tract. Some key issues. In Wing, A., Haggard, P. \& Flanagan, J.R. (Eds), Hand and Brain: the Neurophysiology and Psychology of Hand Movements. Academic Press, San Diego, pp. 125-145. 
Bonnard, M., Camus, M., De Graaf, J. \& Pailhous, J. (2003) Direct evidence for a binding between cognitive and motor functions in humans: a TMS study. J. Cogn. Neurosci., 15, 1207-1216.

Bonnard, M., De Graaf, J. \& Pailhous, J. (2004) Interactions between cognitive and sensorimotor functions in the motor cortex: evidence from the preparatory motor sets anticipating a perturbation. Rev. Neurosci., 15, 371382 .

Davare, M., Andres, M., Cosnard, G., Thonnard, J.L. \& Olivier, E. (2006) Dissociating the role of ventral and dorsal premotor cortex in precision grasping. J. Neurosci., 26, 2260-2268.

Devanne, H., Cohen, L.G., Kouchtir-Devanne, N. \& Capaday, C. (2002) Integrated motor cortical control of task-related muscles during pointing in humans. J. Neurophysiol., 87, 3006-3017.

Dum, R.P. \& Strick, P.L. (2005) Frontal lobe inputs to the digit representations of the motor areas on the lateral surface of the hemisphere. J. Neurosci., 25, 1375-1386.

Evarts, E.V. \& Fromm, C. (1978) The pyramidal tract neuron as summing point in a closed-loop control system in the monkey. In Desmedt, J.E. (Ed.), Motor Control in Man: Long-Loop Mechanisms. Karger, Basel. pp. 56-69.

Fadiga, L., Buccino, G., Craighero, L., Fogassi, L., Gallese, V. \& Pavesi, G. (1999) Corticospinal excitability is specifically modulated by motor imagery: a magnetic stimulation study. Neuropsychologia, 37, 147-158.

Flament, D., Goldsmith, P., Buckley, C.J. \& Lemon, R.N. (1993) Task dependence of responses in first dorsal interosseous muscle to magnetic brain stimulation in man. J. Physiol. (Lond.), 464, 361-378.

Fromm, C. (1987) Sensorimotor integration: the role of pyramidal tract neurons. In Heuer, H. \& Sanders, A.F. (Eds), Perspectives on Perception and Action. Lawrence Erlbaum Associates, Hillsdale, NJ. pp. 131-168.

Galléa, C., De Graaf, J., Bonnard, M. \& Pailhous, J. (2005) High level of dexterity: Differential contributions of frontal and parietal areas. Neuroreport, 16, 1271-1274.

Georgopoulos, A.P. (2000) Neural aspects of cognitive motor control. Curr. Opin. Neurobiol., 10, 238-241.

Geyer, S., Matelli, M., Luppino, G. \& Zilles, K. (2000) Functional neuroanatomy of the primate isocortical motor system. Anat. Embryol. (Berl.), 202, 443-474.

Hasegawa, Y., Kasai, T. \& Tsuji, T. (2001) Further insight into the taskdependant excitability of motor evoked potentials in first dorsal interosseous muscle in humans. Exp. Brain Res., 140, 387-396.

Johansen-Berg, H. \& Matthews, P.M. (2002) Attention to movement modulates activity in sensori-motor areas, including primary motor cortex. Exp. Brain Res., 142, 13-24.

Kristeva-Feige, R., Fritsch, C., Timmer, J. \& Lücking, C.H. (2002) Effects of attention and precision of exerted force on beta range EEG-EMG synchronization during a maintained motor contraction task. Clin. Neurophysiol., 113, 124-131.

Kuhtz-Buschbeck, J.P., Ehrsson, H.H. \& Forssberg, H. (2001) Human brain activity in the control of fine static precision grip forces: an fMRI study. Eur. J. Neurosci., 14, 1-10.
Kuhtz-Buschbeck, J.P., Mahnkopf, C., Holzknecht, C., Siebner, H., Ulmer, S. \& Jansen, O. (2003) Effector-independent representations of simple and complex imagined finger movements: a combined fMRI and TMS study. Eur. J. Neurosci., 18, 3375-3387.

Lemon, R.N., Johansson, R.S. \& Westling, G. (1995) Corticospinal control during reach, grasp, and precision lift in man. J. Neurosci., 15, 6145-6156.

Maier, M.A., Bennett, K.M., Hepp-Reymond, M.C. \& Lemon, R.N. (1993) Contribution of the monkey corticomotoneuronal system to the control of force in precision grip. J. Neurophysiol., 69, 772-785.

Muir, R.B. \& Lemon, R. (1983) Corticospinal neurons with a special role in precision grip. Brain Res., 261, 312-316.

Nakajima, K., Maier, M.A., Kirkwood, P.A. \& Lemon, R.N. (2000) Striking differences in transmission of corticospinal excitation to upper limb motoneurons in two primate species. J. Neurophysiol., 84, 1-12.

Porro, C.A., Francescato, M.P., Cettolo, V., Diamond, M.E., Baraldi, P., Zuiani, C., Bazzocchi, M. \& di Prampero, P.E. (1996) Primary motor and sensory cortex activation during motor performance and motor imagery: a functional magnetic resonance imaging study. J. Neurosci., 16, 76887698.

Porter, R. \& Lemon, R. (1993) Corticospinal Function and Voluntary Movement. Clarendon Press, Oxford.

Requin, J., Brener, J. \& Ring, C. (1991) Preparation for action. In Jennings, J.R. \& Coles, M.G. (Eds), Handbook of Cognitive Psychophysiology: Central and Autonomic Nervous System Approaches. John Wiley \& Sons, New York. pp. $357-448$.

Riehle, A. (2004) Preparation for action: one of the key functions of motor cortex. In Riehle, A. \& Vaadia, E. (Eds), Motor Cortex in Voluntary Movements, a Distributed System for Distributed Functions. CRC Press, Boca Raton, FL. pp. 213-240.

Rizzolatti, G., Luppino, G. \& Matelli, M. (1998) The organization of the cortical motor system: new concepts. Electroencephalogr. Clin. Neurophysiol., 106, 283-296.

Roland, P.E. \& Zilles, K. (1996) Functions and structures of the motor cortices in humans. Curr. Opin. Neurobiol., 6, 773-781.

Rossi, S., Pasqualetti, P., Tecchio, F., Pauri, F. \& Rossini, P.M. (1998) Corticospinal excitability modulation during mental simulation of wrist movements in human subjects. Neurosci. Lett., 243, 147-151.

Schieber, M. (2001) Constraints on somatotopic organization in the primary motor cortex. J. Neurophysiol., 86, 2125-2143.

Schieppati, M., Trompetto, C. \& Abbruzzese, G. (1996) Selective facilitation of responses to cortical stimulation of proximal and distal arm muscles by precision tasks in man. J. Physiol. (Lond.), 491, 551-562.

Tinazzi, M., Farina, S., Tamburin, S., Facchini, S., Fiaschi, A., Restivo, D. \& Berardelli, A. (2003) Task-dependant modulation of excitatory and inhibitory functions within the human primary motor cortex. Exp. Brain Res., 150, 222-229.

Yousry, T.A., Schmid, U.D., Alkadhi, H., Schmidt, D., Peraud, A., Buettner, A. \& Winkler, P. (1997) Localization of the motor hand area to a knob on the precentral gyrus. A new landmark. Brain, 120, 141-157. 